\title{
Diagnosis and Treatment of Pancreas Rejection
}

\author{
R. R. Redfield ${ }^{1}$ • D. B. Kaufman ${ }^{1}$ • J. S. Odorico ${ }^{1}$
}

Published online: 11 April 2015

(C) The Author(s) 2015. This article is published with open access at Springerlink.com

\begin{abstract}
Despite significant improvement in pancreas allograft survival, rejection of the pancreas remains a major clinical problem. In addition to cellular rejection of the pancreas, antibody-mediated rejection of the pancreas is now a welldescribed entity. The 2011 Banff update established comprehensive guidelines for the diagnosis of acute and chronic AMR. The pancreas biopsy is critical in order to accurately diagnose and treat pancreas rejection. Other modes of monitoring pancreas rejection we feel are neither sensitive nor specific enough. In this review, we examine recent advances in the diagnosis and treatment of pancreas rejection as well as describe practical diagnostic and treatment algorithms.
\end{abstract}

Keywords Diagnosis · Treatment $\cdot$ Pancreas rejection

\section{Introduction}

Solid organ pancreas transplant outcomes continue to improve [1], with the half-life of simultaneous pancreas and kidney (SPK) allograft approaching 15 years and that of solitary pancreas recipients nearing 13 years [2]. Much of this success can likely be attributed to improved surgical technique, immunosuppression, and diagnosis and treatment of rejection. In this

This article is part of the Topical Collection on Pancreas Transplantation

J. S. Odorico

jon@surgery.wisc.edu

1 Division of Transplantation, Department of Surgery, University of Wisconsin-Madison School of Medicine and Public Health, University of Wisconsin Hospital and Clinics, 600 Highland Ave, Clinical Science Cntr-H4/756, Madison, WI 53792, USA review, we will specifically focus on recent advances in the diagnosis and treatment of pancreas rejection.

\section{Clinical Presentation of Rejection}

Clinically, pancreas allograft function is commonly monitored by a number of parameters including hyperglycemia, serum amylase, serum lipase, C-peptide level, hemoglobin A1C, or, if bladder drained, urinary amylase. However, in order to accurately identify and define rejection, these clinical tools are insufficient, because they are too non-specific. There are multiple potential causes for hyperamylasemia, hyperlipasemia, and hyperglycemia. Even stable kidney function, or a normal serum creatinine in SPK transplants, is not a consistently reliable indicator that pancreatic graft immunological damage is not occurring [4]. Indeed, it is not uncommon to observe isolated pancreas rejection in the setting of normal kidney allograft function (Table 1).

Elevated pancreatic enzymes is the most common presentation of pancreatic allograft rejection, and most patients are asymptomatic or have only mild graft tenderness upon presentation. If a patient is asymptomatic, the source of the elevated enzymes is more likely to be from the transplanted pancreas as the graft itself is insensate. In contrast, symptomatic increases in pancreatic enzymes can point to either transplant or native gland disease. Lipase is considered to be more specific than amylase and has greater sensitivity in the author's experience. Naturally, these signs and symptoms can arise from multiple causes in the setting of an enterically drained graft. Hence, it is useful to consider the differential diagnosis and the timing of presentation when evaluating these patients (Fig. 1, Table 2). 
Table 1 Pancreas allograft biopsies at the University of Wisconsin from January 1994 to July 2012

\begin{tabular}{ll} 
Total & 422 \\
Percutaneous & $406(96.2 \%)$ \\
Rejection (of any kind) & $265(62.8 \%)$ \\
$\quad$ With normal amylase and lipase & $20(7.5 \%)$ \\
Pancreatitis & $16(3.8 \%)$ \\
Non-diagnostic & $24(5.7 \%)$ \\
Simultaneous pancreas and kidney biopsies & 20 \\
Discordant for rejection & $5(25 \%)$ \\
\hline
\end{tabular}

An algorithm for the diagnosis and management of patients with increased pancreatic enzymes is shown in Fig. 2 [3•]. In our practice, the initial approach to the patient with elevated enzymes is history and physical, fasting C-peptide, $\mathrm{HbAlC}$, donor-specific antibodies (DSA), and an imaging study, preferably CT scan of the abdomen and pelvis with IV and PO contrast (Fig. 2). In the perioperative period ( $<45$ days) or in the presence of abdominal symptoms, it is important to obtain a CT scan first before allograft biopsy to evaluate for postsurgical complications or intra-abdominal infection. On the other hand, if the patient is beyond the perioperative period and without abdominal symptoms, a surgical cause for the elevated enzymes is significantly less likely, and therefore, it is reasonable to go straight to pancreas allograft biopsy and assess the graft for anatomical abnormalities during performance of the biopsy. Regardless of the time frame posttransplant, a pancreas allograft biopsy and CT scan will efficiently identify the cause for elevated pancreatic enzymes in the vast majority of cases.
Medication non-compliance, recent intolerance of oral medications, alcohol consumption, recent abdominal trauma, constipation, presence of a ventral hernia, abdominal pain, nausea, vomiting, and obstructive symptoms are important points to elicit from the patient. Duodenal or parenchymal enzyme leak, pseudocyst, mycotic aneurysm, SBO, intrinsic pancreatic abnormalities such as duct strictures, and tumors are included in the differential diagnosis of elevated enzymes and are usually diagnosed with imaging studies.

In our experience, transplant pancreatitis that is not attributable to an anatomic or immunological cause is very uncommon. Instead, transplant pancreatitis, putatively defined as increased pancreatic enzymes without evidence of other anatomic abnormality on CT scan other than peri-pancreatic transplant inflammation and graft swelling, can also be indicative of acute or chronic rejection or CMV pancreatitis. In our large series of for-cause pancreas allograft biopsies, non-immunological pancreatitis was only diagnosed on biopsy in $4 \%(16 / 422)$ of biopsies, a frequency similar to that reported previously [4]. Therefore, transplant pancreatitis should be considered a diagnosis of exclusion (Table 1).

While most patients who have acute rejection present with elevated pancreatic enzymes, it is not uncommon to see patients develop chronic rejection and graft failure without significant increases in their serum enzymes. Why chronic rejection does not always coincide with serological evidence of acinar injury is not presently known, but may be related to progressive loss of acinar/parenchymal tissue resulting in less release of enzymes and/or reduced lab frequency resulting in missed detection.

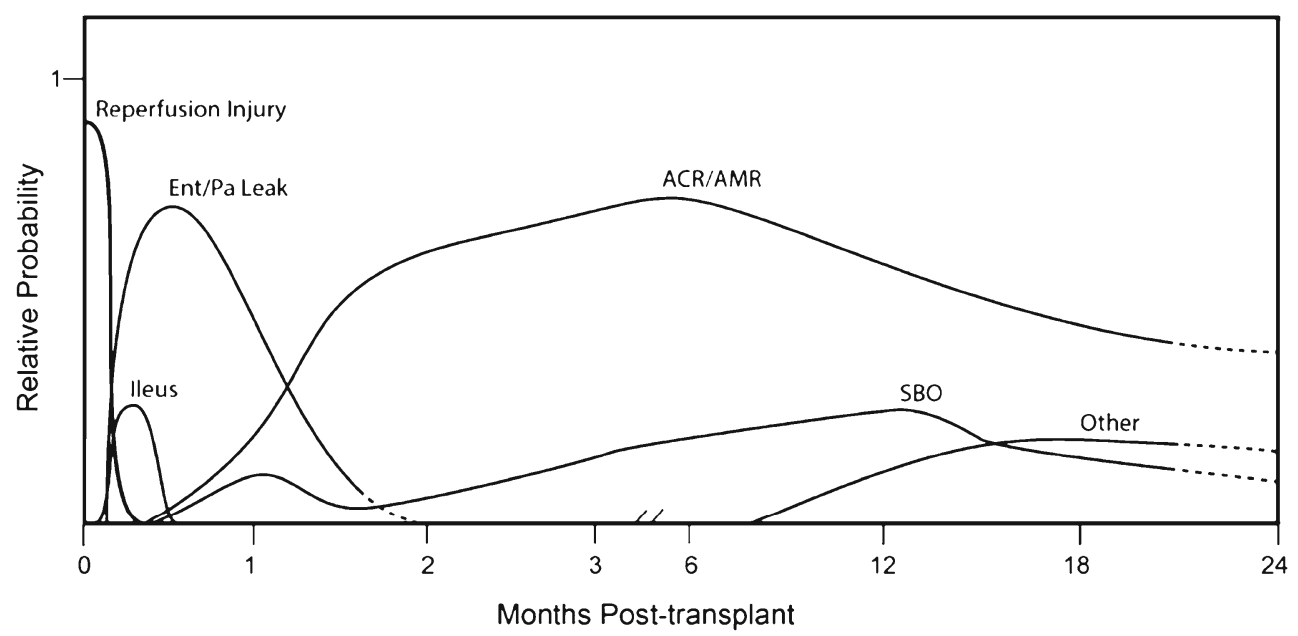

Fig. 1 Temporal relationship of elevated pancreas enzymes to etiology. Surgical complications typically present early in the postoperative course, whereas rejection most commonly presents later. "Other" includes possible causes such as transplant pancreatic duct stricture, native pancreatitis, IPMN or cancer in pancreas transplant or native pancreas, and penetrating ulcer. Of note, although complete graft thrombosis can present as increased pancreatic enzymes, in our experience, this is a very uncommon presentation, and when most grafts thrombose, very limited increases in enzymes are seen if at all. Relative probability on the $Y$-axis does not represent the overall probability or incidence of this complication; instead, it strives to convey the relative probability that these diagnoses are associated with elevated pancreatic enzymes. Abbreviations: $A C R / A M R$ acute cellular rejection/antibody-mediated rejection, Ent/Pa Leak enteric or pancreatic leak, $S B O$ small bowel obstruction 
Table 2 Common diagnoses contributing to increased pancreatic enzymes after pancreas transplantation

\begin{tabular}{|c|c|c|}
\hline Diagnosis & Overall frequency $(\%)$ & $\begin{array}{l}\text { Frequency the entity presents } \\
\text { as increased enzymes }\end{array}$ \\
\hline \multicolumn{3}{|l|}{ Perioperative ( $<45$ days) } \\
\hline Enzyme leak $^{1}$ & $\sim 5$ & High \\
\hline Infected fluid/abscess & $\sim 5$ & Moderate \\
\hline Thrombosis & $2-5$ & Low \\
\hline Ileus & $5-10$, transient & Moderate \\
\hline Acute rejection & $1-2$ & High \\
\hline \multicolumn{3}{|l|}{ Mid postoperative ( $>45$ days -1 year) } \\
\hline Acute rejection $^{\mathrm{a}}$ & $15-20$ & High \\
\hline SBO & 5 & Low-moderate \\
\hline Pseudocyst & $2-5$ & High \\
\hline Constipation & $2-5$ & Low-moderate \\
\hline Abscess & $2-5$ & Low-moderate \\
\hline CMV pancreatitis & $\sim 1$ & High \\
\hline \multicolumn{3}{|l|}{ Late postoperative ( $>1$ year) } \\
\hline Acute rejection & $5-10$ & High \\
\hline Chronic rejection & $\sim 5$ & Moderate-high \\
\hline $\mathrm{SBO} /$ ventral hernia & $\sim 10$ & Low-moderate \\
\hline Intrinsic pancreatic abnormality ${ }^{\mathrm{b}}$ & $\sim 5$ & Moderate-high \\
\hline Native pancreatitis & 1 & High \\
\hline CMV pancreatitis & $<1$ & High \\
\hline
\end{tabular}

${ }^{1}$ Enzyme leak - enteric or parenchymal, including pseudocyst or pancreatic ascites

${ }^{a}$ Frequency depends on risk factors such as type of transplant, primary vs. re-transplant, sensitized status, donorspecific antibody, race mismatch, and donor age

${ }^{\mathrm{b}}$ Includes graft trauma, chemical pancreatitis, pancreatic duct stricture, IPMN, carcinoma, pseudocyst

Abbreviations: $S B O$ small bowel obstruction, $C M V$ cytomegalovirus

\section{Pancreas Allograft Biopsy}

In order to diagnose pancreas rejection accurately, a biopsy of the pancreas allograft is necessary. It is currently the only available means of grading the severity of rejection and distinguishing antibody-mediated rejection (AMR) from acute cellular rejection (ACR). The most commonly performed approach to allograft biopsy is percutaneous ultrasound-guided biopsy of the pancreas parenchyma, which can be performed in either bladder-drained or enterically drained allografts safely and effectively [5,6]. Alternative methods of acquiring tissue from the pancreaticoduodenal allograft have been described. These include percutaneous CT-guided biopsy, endoscopic biopsy of the donor duodenum for enterically drained pancreata, or, if bladder drained, cystoscopic needle biopsy of pancreatic head and/or mucosal biopsy of the donor duodenum. The potential for discordant findings between pancreas histology and duodenal histology [7-9] has limited the widespread reliance of sampling duodenal mucosa for diagnosis. Furthermore, C4d staining features characteristic of AMR in the duodenal mucosa have not been adequately defined, and current methods for staining duodenal mucosal biopsies for
C4d may not be sufficiently specific. Additionally, using the kidney as a sentinel organ, as can be done in SPK transplants, can be misleading. In our experience, $\sim 25 \%$ of kidney and pancreas biopsies in SPK patients are discordant (Table 1). Prior studies have demonstrated that isolated pancreas rejection with normal renal function in SPK patients is not uncommon $[4,10]$. Therefore, we advocate for an actual core biopsy of pancreatic tissue to rule out rejection.

In our practice, we prefer ultrasound-guided biopsy with an 18-gauge automatic biopsy device. Our trajectory for biopsy is ideally towards the tail, avoiding the splenic artery and vein. Because of the nest of vessels and larger duct in the head region, we prefer to biopsy the body/tail of the graft aiming either transversely or preferably longitudinally with respect to the axis of the pancreas. We have performed 406 percutaneous biopsies since 1992, with a very low complication rate and no graft losses. Two patients required reoperation: one for bleeding and one for evacuation of pancreatic ascites, which ultimately resolved, and both patients currently still retain excellent graft function. If we are unsuccessful, or there is no suitable safe window free of overlying bowel, then we proceed with CT-guided 
biopsy using a posterior approach, or alternatively an open or laparoscopic biopsy, but this is required rarely. The majority of pancreas biopsies were diagnostic, only $6 \%(24 /$ 422) were non-diagnostic (Table 1).

\section{Distinguishing Histologic Features of AMR, ACR, and Pancreatitis}

The 2007 Banff guidelines for the diagnosis of pancreas rejection solidified prior histopathological descriptions and focused on ACR [11-13]. However, in response to reports in the literature documenting pancreas AMR, the 2011 Banff update established comprehensive guidelines for diagnosis of acute and chronic AMR [14] of the pancreas (Table 3). While a complete review of current histopathological criteria of rejection is beyond the scope of this review, suffice it to say that the only definitive way to precisely diagnosis the severity and mechanism of pancreas allograft rejection is with a pancreas biopsy. In our series of 422 for-cause biopsies, $63 \%$ were diagnostic for rejection; only $4 \%$ were determined to be graft pancreatitis (Table 1).

\section{Incidence and Risk Factors for Rejection}

Niederhaus et al. recently reported on the frequency of both cellular and antibody-mediated pancreas allograft rejection as they relate to specific risk factors [15••]. The incidence of rejection within 1-year posttransplant in a cohort of 162 patients of all pancreas transplant types including many retransplants was $21 \%$, with antibody-mediated rejection (AMR), acute cellular rejection (ACR), and mixed rejection occurring in nearly equal frequency. In their study, the majority of pancreas rejection episodes were successfully reversed and graft function was maintained; however, $20 \%$ of grafts were lost within a year of diagnosis.

Dong et al. similarly showed a 1 -year acute rejection rate of $14.7 \%$ increasing to $26.6 \%$ by 5 years posttransplant [16]. The findings of Dong et al. also support the notion that allograft rejection even if diagnosed and treated aggressively is associated with pancreas graft failure in a subset of patients especially if diagnosed beyond 3 months posttransplant [11].

Risk factors for rejection identified in these two studies include non-primary SPK transplants, primary solitary pancreas alone (PTA), race mismatch [10], and increasing donor age $[15 \bullet \bullet, 16]$. Increased vigilance for rejection in these scenarios,

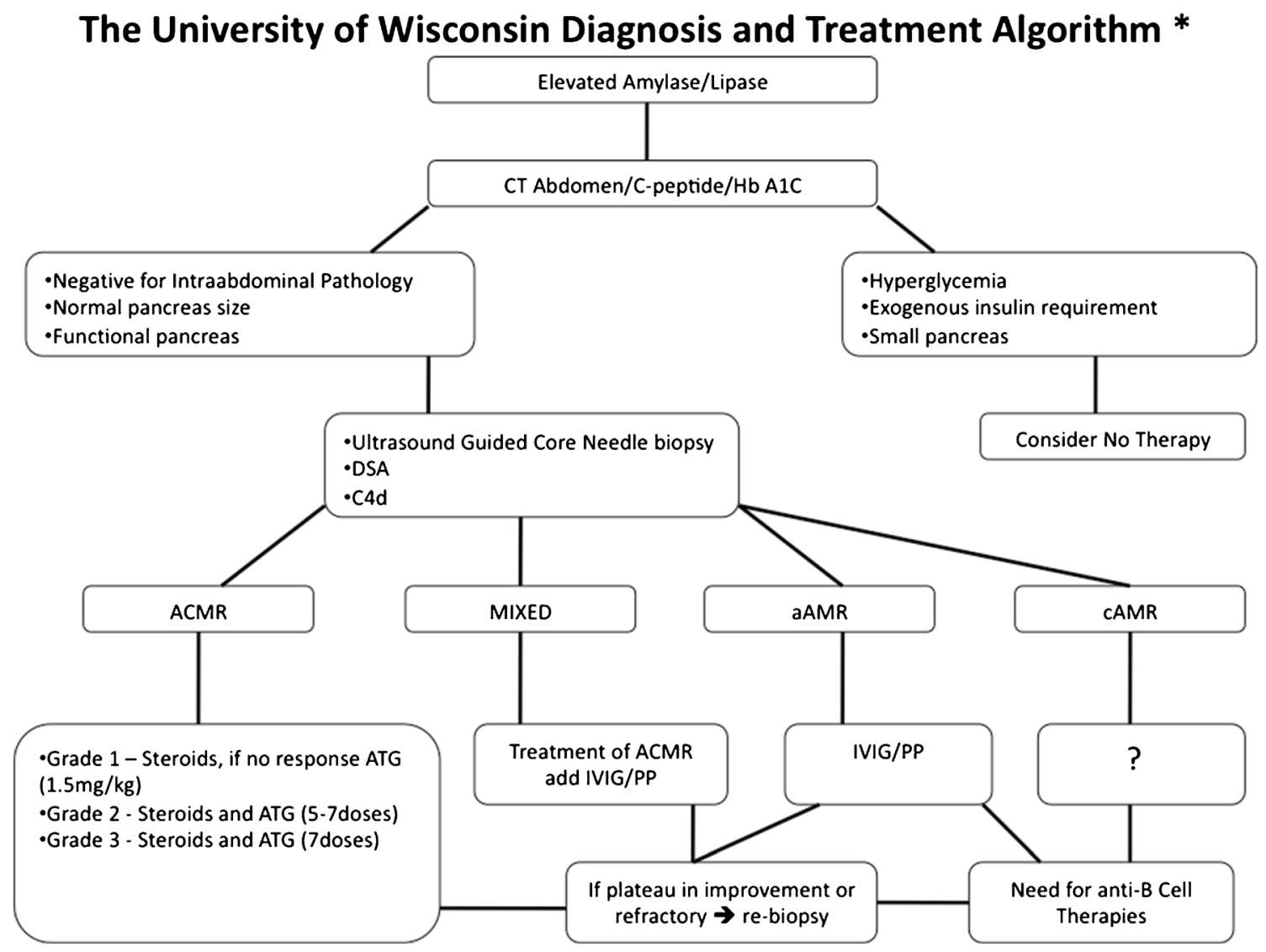

Fig. 2 The University of Wisconsin Diagnosis and Treatment Algorithm Abbreviations: DSA donor-specific antibody, $A C M R$ acute cell-mediated rejection, $A A M R$ acute antibody-mediated rejection, $C A M R$ chronic antibody-mediated rejection, $A T G$ anti-thymocyte globulin, $I V I g$ intravenous immunoglobulin, $P P$ plasmapheresis. [(Published in Trends in Transplantation, (C) Permanyer Publications) 2]. 
Table 3 Banff 2011 classification of cell- and antibody-mediated rejection of the pancreas

\section{Acute $\mathrm{T}$ cell-mediated rejection}

Grade I/mild acute T cell-mediated rejection - active septal inflammation (activated, blastic lymphocytes, and \pm eosinophils) involving septal structures: venulitis (subendothelial accumulation of inflammatory cells and endothelial damage in septal veins, ductitis (epithelial inflammation and damage of ducts)

and/or

Focal acinar inflammation. No more than two inflammatory foci per lobule with absent or minimal acinar cell injury

Grade II/moderate acute T cell-mediated rejection (requires differentiation from AMR)

Multifocal (but not confluent or diffuse) acinar inflammation ( $\geq 3$ foci per lobule) with spotty (individual) acinar cell injury and dropout

and/or

Mild intimal arteritis (with minimal, $<25 \%$ luminal compromise)

Grade III/severe acute T cell-mediated rejection (requires differentiation from AMR)

Diffuse (widespread, extensive) acinar inflammation with focal or diffuse multicellular/confluent acinar cell necrosis and/or moderate or severe intimal arteritis, $>25 \%$ luminal compromise

and/or

Transmural inflammation - necrotizing arteritis

2. Antibody-mediated rejection

(a) Confirmed circulating donor-specific antibody (DSA)

(b) Morphological evidence of tissue injury (interacinar inflammation/ capillaritis, acinar cell damage, swelling/necrosis/apoptosis/dropout, vasculitis, thrombosis)

(c) $\mathrm{C} 4 \mathrm{~d}$ positivity in interacinar capillaries (IAC, $\geq 5 \%$ of acinar lobular surface)

Acute AMR 3 of 3 diagnostic components

Consistent with acute AMR 2 of 3 diagnostic components

Requires exclusion of AMR 1 of 3 diagnostic components

See below for histological grading of acute AMR

Chronic active antibody-mediated rejection: combined features of categories 1 and 2 in the absence of features of ACMR

3. Chronic allograft rejection/graft fibrosis

Stage I (mild graft fibrosis)

Expansion of fibrous septa; the fibrosis occupies less than $30 \%$ of the core surface but the acinar lobules have eroded, irregular contours. The central lobular areas are normal

Stage II (moderate graft fibrosis)

The fibrosis occupies $30-60 \%$ of the core surface. The exocrine atrophy affects the majority of the lobules in their periphery (irregular contours) and in their central areas (thin fibrous strands crisscross between individual acini)

Stage III (severe graft fibrosis)

The fibrotic areas predominate and occupy more than $60 \%$ of the core surface with only isolated areas of residual acinar tissue and/or islets present

Histological grading of acute AMR

Grade I/mild acute AMR

Well-preserved architecture, mild monocytic-macrophagic or mixed (monocytic-macrophagic/neutrophilic) infiltrates with rare acinar cell damage

Grade II/moderate acute AMR

Overall preservation of the architecture with interacinar monocyticmacrophagic or mixed (monocytic-macrophagic/neutrophilic) infiltrates, capillary dilatation, capillaritis, congestion, multicellular acinar cell dropout, and extravasation of red blood cells
Table 3 (continued)

Grade III/severe acute AMR

Architectural disarray, scattered inflammatory infiltrates in a background of interstitial hemorrhage, multifocal and confluent parenchymal necrosis, arterial and venous wall necrosis, and thrombosis

(With permission from: Drachenberg CB, Torrealba JR, Nankivell BJ, Rangel EB, Bajema IM, Kim DU, et al. Guidelines for the diagnosis of antibody-mediated rejection in pancreas allografts - updated Banff grading schema. American Journal of Transplantation. 2011;11 (9):1792802)]]

which are associated with higher immunological risk, may therefore be warranted.

\section{Donor-Specific Antibody as a Biomarker of Rejection}

The deleterious effects of both pre-formed donor-specific antibodies (DSA) and de novo DSA are well established in the kidney transplant literature [17]. Few studies, however, have directly evaluated the role of DSA in pancreas transplantation. Cantarovich et al. showed that DSA in SPK transplants was an independent predictor of graft failure. Of their patients, $24 \%$ (40/167, 152 were SPK recipients) were found to have DSA postoperatively. Maintenance therapy was with antithymocyte globulin, tacrolimus, and mycophenolate mofetil only. This study identified DSA as an independent predictor of graft failure [18]. However, DSA was not quantified in all patients preoperatively so it is unclear from this study what proportion of recipients developed de novo DSA vs. those who already had pre-formed DSA.

Mittal et al. demonstrated in a large cohort of pancreas transplant patients that de novo DSA was also an independent risk factor for graft loss [19•]. In this study of 439 pancreas transplant patients (73\% SPK), de novo DSA developed in $37.9 \%$ of patients of the patients who had follow-up. The DSA MFI cutoff was 1000. Pancreas biopsies in association with elevated DSA were not reported in this study. The immunosuppressive regimen consisted of alemtuzumab induction followed by MMF and tacrolimus maintenance immunosuppression. Steroids were not used as maintenance therapy. This is a surprisingly high rate of de novo DSA, and one wonders if it was causally associated with this alemtuzumab/steroid-free immunosuppressive regimen. There are other reports of alemtuzumab associated with increased DSA production in kidney transplant recipients, which may explain such an elevated incidence of de novo DSA [20]. Obviously, this area requires further study to understand the exact mechanisms of these observations, especially the association and timing of de novo DSA in relation to histopathological rejection. But nonetheless, strategies to prevent or reduce de novo DSA may lead to better graft outcomes. 
In our practice, the presence of pretransplant DSA aids our assessment of the posttransplant immunologic risk. For patients with pretransplant DSA and a negative flow crossmatch, we tend to favor using a depleting antibody for induction therapy. We monitor all our patients with DSA measurements postoperatively with the frequency of monitoring determined by a preoperative risk stratification. However, it is currently unknown what to do with de novo DSA in the setting of normal pancreas allograft function. One hypothesis is that it indicates under immunosuppression, and increasing immunosuppression maybe warranted. Elevated DSA in the setting of normal graft function could also be a harbinger of eventual graft dysfunction or simply could be of no clinical consequence. Certainly more studies are needed. If the emergence or rise in DSA does in fact accompany abnormal graft function, then a biopsy is warranted to rule out rejection.

\section{Treatment of Pancreas Rejection}

A normal fasting C-peptide and $\mathrm{HbAlc}$ confirms a functioning pancreas allograft prior to the initiation of anti-rejection therapy. Consideration of pancreas graft functional reserve prior to initiating any anti-rejection therapy is paramount. Hyperglycemia, as evidenced by significantly elevated fasting blood glucose or $\mathrm{HbA1c}$, or low fasting C-peptide level should be taken into account when weighing the potential benefits and risks of pursuing therapy. Additionally, a significant chronic component to the patient's rejection status, as evidenced by fibrotic changes on biopsy, should factor into the potential benefit of therapy. Treatment of chronic or acute rejection if significant hyperglycemia is present prior to initiation of steroid therapy, or if the patient is on insulin, is controversial, but traditionally, hyperglycemia had been thought to be a marker of poor salvagability in a pancreas allograft [12]. If the patient's graft dysfunction has progressed to the point of requiring large doses of exogenous insulin, forgoing treatment for rejection should be considered, especially since several therapeutic agents will only exacerbate hyperglycemia. If the graft has evidence of significant fibrosis, and/or a paucity of islets and/or is small on imaging, and C-peptide is low, then chronic rejection is probable and little will be gained with treatment, or until at least better therapies are developed. On the other hand, if the patient has only minimal fibrosis and primarily acute changes, with detectable normal appearing islets, imaging that shows a normal-sized gland, and fasting C-peptide is easily detectable well into the normal range, then it is worth treating the rejection process aggressively in our experience even with the short-term prospect of worsening glycemic control and temporary insulin use.

H\&E staining can diagnose and grade ACR, and we treat accordingly with steroids $+/-$ anti-thymocyte globulin (ATG) as illustrated in Fig. 2. It is important to note that, if ACR is present, it should be treated aggressively. In the setting of mixed cellular and AMR, we recommend that initial therapy be directed against the cellular component. Niederhaus et al. found, even in cases of mixed rejection, the pancreas graft survival mirrored that of ACR alone if treated [15••]. The incidence of mixed rejection is not trivial, occurring in $7 \%$ of recipients at 1 year. At this time, it is unclear if mixed rejection requires IVIG, plasmapheresis, and/or specific antiB cell therapies or if steroids and ATG is sufficient. If the biopsy is negative or indeterminate for ACR, we await results of C4d immunostaining and DSA, which usually takes a day or two longer in our laboratory.

If DSA is positive with histologic findings consistent with AMR, even in the absence of $\mathrm{C} 4 \mathrm{~d}$, we proceed with treatment consisting of IVIG and plasmapheresis as we would in the setting of significant $\mathrm{C} 4 \mathrm{~d}$ staining. In cases of a mixed rejection, we generally add ATG. If the amylase/lipase decline, we continue treatment until they have normalized. If their descent stalls or if they start to rise again, we usually repeat the biopsy. If the re-biopsy demonstrates persistent AMR, additional antiB cell therapies, such as bortezomib, rituximab, or eculizumab, are considered. Admittedly, treatment of pancreas AMR is currently pursued without clear data and the aforementioned approach is merely proposed as a guide. Even in the kidney AMR literature, there is a paucity of randomized clinical trails. Despite this, there have been advances in the treatment of AMR resulting in improved outcomes. The main treatment goal in AMR is for the reduction of DSA and elimination of the $\mathrm{B}$ cell and plasma cell population responsible for the production of DSA. Hopefully, carefully protocolized treatments and the examination of outcomes will be prospectively conducted.

Overall, key points of our treatment algorithm (Fig. 2) are as follows: ensure the pancreas is still functioning and therefore worthy of salvage, obtain tissue diagnosis to evaluate the degree of ACR and AMR, and treat ACR aggressively as a first and primary strategy. Sequentially, escalate therapy as data becomes available and/or if there is no improvement in enzymes with ACR-directed treatment. Finally, if there is any doubt as to the cause of ongoing enzyme elevation or hyperglycemia, it is valuable to consider re-biopsy. While we acknowledge this field is still evolving, we believe the aforementioned approach is a reasonable management protocol (Fig. 2).

\section{Conclusions}

In our practice of evaluating each patient for the possibility of pancreas graft rejection, we gather serologic and histologic data in the form of DSA and biopsy, and if hyperglycemic, then fasting $\mathrm{C}$-peptide and $\mathrm{HbA} 1 \mathrm{c}$. Once a diagnosis of rejection is made, we treat accordingly as described in Fig. 2 for 
ACR, AMR, and mixed types of rejections, as long as the pancreas is worth saving (i.e., no evidence of hyperglycemia or elevated $\mathrm{HbAl} \mathrm{c})$. One strategy is to use currently available anti-T and anti-B cell-directed therapies sequentially, progressively escalating therapies over the course of several days to a week, depending on the response and relative contribution of each component to the active rejection process. However, it should also be kept in mind that clear data is lacking in this area. Moving forward, more rigorous study is required to define the best treatment regimens for rejection.

Acknowledgments The authors would like to thank Rebecca Wasieleski for her assistance with the figures and Bridget Welch and Cheryl Bowman for their assistance with our pancreas biopsy data.

\section{Compliance with Ethics Guidelines}

Conflict of Interest RR Redfield, DB Kaufman, and JS Odorico declare that they have no conflict of interest.

Human and Animal Rights and Informed Consent This article does not contain any studies with human or animal subjects performed by any of the authors.

Open Access This article is distributed under the terms of the Creative Commons Attribution 4.0 International License (http:// creativecommons.org/licenses/by/4.0/), which permits unrestricted use, distribution, and reproduction in any medium, provided you give appropriate credit to the original author(s) and the source, provide a link to the Creative Commons license, and indicate if changes were made.

\section{References}

Papers of particular interest, published recently, have been highlighted as:

- Of importance

•. Of major importance

1. Kandaswamy R, Stock PG, Skeans MA, Gustafson SK, Sleeman EF, Wainright JL, et al. OPTN/SRTR 2011 annual data report: pancreas. Am J Transplant: Off J Am Soc Transplant Am Soc Transplant Surg. 2013;1(13 Suppl):47-72.

2. Gruessner RW, Gruessner AC. The current state of pancreas transplantation. Nat Rev Endocrinol. 2013;9(9):555-62.

3. Redfield RR, McCune KR, Sollinger HW, Odorico JS. Pancreas allograft antibody mediated rejection: current concepts and future therapies. Trends Transplan. 2014; In Press.Very indepth and uptodate review of $A M R$.

4. Klassen DK, Hoen-Saric EW, Weir MR, Papadimitriou JC, Drachenberg CB, Johnson L, et al. Isolated pancreas rejection in combined kidney pancreas tranplantation. Transplantation. 1996;61(6):974-7.

5. Allen RD, Wilson TG, Grierson JM, Greenberg ML, Earl MJ, Nankivell BJ, et al. Percutaneous biopsy of bladder-drained pancreas transplants. Transplantation. 1991;51(6):1213-6.
6. Klassen DK, Weir MR, Cangro CB, Bartlett ST, Papadimitriou JC, Drachenberg CB. Pancreas allograft biopsy: safety of percutaneous biopsy-results of a large experience. Transplantation. 2002;73(4): 553-5.

7. Laftavi MR, Gruessner AC, Bland BJ, Foshager M, Walsh JW, Sutherland DE, et al. Diagnosis of pancreas rejection: cystoscopic transduodenal versus percutaneous computed tomography scanguided biopsy. Transplantation. 1998;65(4):528-32.

8. de Kort H, Roufosse C, Bajema IM, Drachenberg CB. Pancreas transplantation, antibodies and rejection: where do we stand? Curr Opin Organ Transplant. 2013;18(3):337-44.

9. Nakhleh RE, Benedetti E, Gruessner A, Troppmann C, Goswitz JJ, Sutherland DE, et al. Cystoscopic biopsies in pancreaticoduodenal transplantation. Are duodenal biopsies indicative of pancreas dysfunction? Transplantation. 1995;60(6):541-6.

10. Klassen DK, Weir MR, Schweitzer EJ, Bartlett ST. Isolated pancreas rejection in combined kidney-pancreas transplantation: results of percutaneous pancreas biopsy. Transplant Proc. 1995;27(1):13334.

11. Drachenberg CB, Odorico J, Demetris AJ, Arend L, Bajema IM, Bruijn JA, et al. Banff schema for grading pancreas allograft rejection: working proposal by a multi-disciplinary international consensus panel. Am J Transplant. 2008;8(6):1237-49.

12. Nakhleh RE, Sutherland DE. Pancreas rejection. Significance of histopathologic findings with implications for classification of rejection. Am J Surg Pathol. 1992;16(11):1098-107.

13. Drachenberg CB, Papadimitriou JC, Klassen DK, Racusen LC, Hoehn-Saric EW, Weir MR, et al. Evaluation of pancreas transplant needle biopsy: reproducibility and revision of histologic grading system. Transplantation. 1997;63(11):1579-86.

14. Drachenberg CB, Torrealba JR, Nankivell BJ, Rangel EB, Bajema IM, Kim DU, et al. Guidelines for the diagnosis of antibodymediated rejection in pancreas allografts-updated Banff grading schema. Am J Transplant. 2011;11(9):1792-802.

15.・ Niederhaus SV, Leverson GE, Lorentzen DF, Robillard DJ, Sollinger HW, Pirsch JD, et al. Acute cellular and antibody-mediated rejection of the pancreas allograft: incidence, risk factors and outcomes. Am J Transplant. 2013;13(11):2945-55. In this work, the authors describe rejection after pancreas transplantation from a large center. AMR occurs at a measurable rate after pancreas transplantation, and the diagnosis should be actively sought.

16. Dong M, Parsaik AK, Kremers W, Sun A, Dean P, Prieto M, et al. Acute pancreas allograft rejection is associated with increased risk of graft failure in pancreas transplantation. Am J Transplant. 2013;13(4):1019-25.

17. Mohan S, Palanisamy A, Tsapepas D, Tanriover B, Crew RJ, Dube $\mathrm{G}$, et al. Donor-specific antibodies adversely affect kidney allograft outcomes. J Am Soc Nephrol: JASN. 2012;23(12):2061-71.

18. Cantarovich D, De Amicis S, Akl A, Devys A, Vistoli F, Karam G, et al. Posttransplant donor-specific anti-HLA antibodies negatively impact pancreas transplantation outcome. Am J Transplant. 2011;11(12):2737-46.

19. Mittal S, Page SL, Friend PJ, Sharples EJ, Fuggle SV. De novo donor-specific HLA antibodies: biomarkers of pancreas transplant failure. Am J Transplant. 2014;14(7):1664-71. Study demonstrating the detrimental effects of DSA on pancreas allograft outcomes.

20. Todeschini M, Cortinovis M, Perico N, Poli F, Innocente A, Cavinato RA, et al. In kidney transplant patients, alemtuzumab but not basiliximab/low-dose rabbit anti-thymocyte globulin induces B cell depletion and regeneration, which associates with a high incidence of de novo donor-specific anti-HLA antibody development. J Immunol. 2013;191(5):2818-28. 